\title{
Auditory processing during deep propofol sedation and recovery from unconsciousness
}

\author{
Stefan Koelsch ${ }^{\mathrm{a}, *}$, Wolfgang Heinke ${ }^{\mathrm{b}, * *}$, Daniela Sammler ${ }^{\mathrm{a}}$, Derk Olthoff ${ }^{\mathrm{b}}$ \\ ${ }^{a}$ Max Planck Institute for Human Cognitive and Brain Sciences, Independent Junior Research Group Neurocognition of Music, \\ Stephanstr 1a, 04103 Leipzig, Germany \\ ${ }^{\mathrm{b}}$ Department of Anesthesiology and Intensive Care Therapy, University of Leipzig, Leipzig, Germany
}

Accepted 21 May 2006

\begin{abstract}
Objective: Using evoked potentials, this study investigated effects of deep propofol sedation, and effects of recovery from unconsciousness, on the processing of auditory information with stimuli suited to elicit a physical MMN, and a (music-syntactic) ERAN.

Methods: Levels of sedation were assessed using the Bispectral Index (BIS) and the Modified Observer's Assessment of Alertness and Sedation Scale (MOAAS). EEG-measurements were performed during wakefulness, deep propofol sedation (MOAAS 2-3, mean BIS =68), and a recovery period. Between deep sedation and recovery period, the infusion rate of propofol was increased to achieve unconsciousness (MOAAS 0-1, mean BIS =35); EEG measurements of recovery period were performed after subjects regained consciousness.

Results: During deep sedation, the physical MMN was markedly reduced, but still significant. No ERAN was observed in this level. A clear P3a was elicited during deep sedation by those deviants, which were task-relevant during the awake state. As soon as subjects regained consciousness during the recovery period, a normal MMN was elicited. By contrast, the P3a was absent in the recovery period, and the P3b was markedly reduced.

Conclusions: Results indicate that the auditory sensory memory (as indexed by the physical MMN) is still active, although strongly reduced, during deep sedation (MOAAS 2-3). The presence of the P3a indicates that attention-related processes are still operating during this level. Processes of syntactic analysis appear to be abolished during deep sedation. After propofol-induced anesthesia, the auditory sensory memory appears to operate normal as soon as subjects regain consciousness, whereas the attention-related processes indexed by P3a and P3b are markedly impaired.

Significance: Results inform about effects of sedative drugs on auditory and attention-related mechanisms. The findings are important because these mechanisms are prerequisites for auditory awareness, auditory learning and memory, as well as language perception during anesthesia.
\end{abstract}

(C) 2006 International Federation of Clinical Neurophysiology. Published by Elsevier Ireland Ltd. All rights reserved.

Keywords: Propofol; Anesthesia; Auditory processing; MMN; P3a; P3b; Syntax

\section{Introduction}

This study aimed at investigating effects of propofol sedation on two components of the event-related brain potential (ERP): the mismatch negativity $(\mathrm{MMN})$ and the early right anterior negativity (ERAN). The MMN can be

* Corresponding authors. Tel.: +49341355 217 11; fax: +49341355 21730 .

E-mail addresses: koelsch@cbs.mpg.de (S. Koelsch), mail@stefankoelsch.de (S. Koelsch), heiw@medizin.uni-leipzig.de (W. Heinke). elicited by physically deviant tones in a series of standard tones and is taken to reflect operations of the auditory sensory memory (Näätänen, 2001; Schröger, 1998). The MMN is generated mainly in, and in the close vicinity of the primary auditory cortex, although neural mechanisms located in the frontal cortex have also reported to be important for the generation of the MMN (Alain et al., 1998; Alho et al., 1994; Doeller et al., 2003; Opitz et al., 2002; Rinne et al., 2000).

The ERAN can be elicited within musical chord sequences by music-structurally irregular chord functions 
and is taken to reflect music-syntactic processing (Koelsch, 2005; Koelsch and Siebel, 2005). It has been shown that the ERAN receives its main contributions from neural generators located in the inferior frontolateral cortex (inferior Brodmann's area 44, Maess et al., 2001), presumably with additional contributions from the anterior portion of the superior temporal gyrus (STG, e.g. Koelsch, 2005). The neural resources of syntax processing in music, thus, overlap with those of syntactic language processing (Janata and Grafton, 2003; Koelsch, 2005; Patel, 2003; Patel et al., 1998). There are several findings reflecting this overlap: (a) The ERAN interacts with the left anterior negativity (LAN, elicited by morpho-syntactic violations, Koelsch et al., 2005b), (b) the ERAN is reminiscent in timecourse and neural generators to the early left anterior negativity (ELAN, elicited by phrase structure violations, e.g. Friederici, 2002), and (c) aphasic patients also show impairment of music-syntactic processing (Patel, 2005). Notably, it is therefore highly likely that, if an ERAN can be elicited under a certain level of sedation, not only musicsyntactic, but also language-syntactic processing is still at work. The question about which auditory processes are still functioning under different levels of sedation is not only relevant to understand the exact effects of sedative drugs on the brain, but also important to gather knowledge about the neural mechanisms underlying auditory perception and language processing during anesthesia. In contrast to the ELAN and the LAN, reliable measures of the ERAN are easier to obtain, mainly because a large amount of trials can be collected in a relatively short time.

It is important to note that the ERAN also resembles the classical MMN elicited by physical deviants: both components (a) have maximal negative polarity at frontal leads, (b) show polarity inversion at mastoidal sites when nose reference is used, (c) have latencies around $100-200 \mathrm{~ms}$, and (d) are elicited by irregular auditory information. However, as mentioned above, the MMN originates predominantly from auditory cortices and reflects operations of the auditory sensory memory, whereas the ERAN mainly originates from frontal cortices and reflects higher-order processing of complex, (music-) syntactic analysis. The use of both MMN and ERAN in studies investigating effects of sedative drugs, thus, allows to assess differential effects of a drug on different cognitive processes originating from different cerebral structures (Heinke and Koelsch, 2005; Heinke et al., 2004b).

There is converging evidence that the MMN is absent under adequate anesthesia (Heinke et al., 2004b; Simpson et al., 2002), and a recent study reported the same for the ERAN (Heinke et al., 2004b). Effects of deep propofol sedation on the neural mechanisms underlying the generation of MMN and ERAN have remained to be specified. Deep sedation reflects a behavioral state in which a person does, e.g. not respond to verbal commands uttered with normal loudness. In contrast to adequate anesthesia, the subject is arouseable during this state by loud, or repeated verbal commands, or by a painful stimulus (similar as during natural sleep). The level of sedation can be assessed by means of clinical scores, for example, the modified observer's assessment of alertness/sedation scale (MOAAS, Chernik et al., 1990, see Table 1), or by means of derived EEG parameters, such as the bispectral index (BIS, Johansen and Sebel, 2000; Rosow and Manberg, 2001). Both techniques are well established to adjust anesthesia at a certain depth.

Studies investigating the effects of deep propofol sedation on the MMN (Heinke et al., 2004b; Simpson et al., 2002; Yppärilla et al., 2002) have not yet provided a consistent picture. Yppärilla et al. (2002) showed an MMN in patients who did not respond to loud auditory stimuli or light tactile stimulation (Ramsey Score 6). Simpson et al. (2002) reported that MMN potentials observed in unresponsive subjects were statistically not significant, and MMN potentials elicited when subjects responded to commands were not consistent (a significant MMN was elicited by a frequency, but not by a duration deviance).

Heinke et al. (2004b) observed both MMN and ERAN in deeply sedated subjects (target plasma concentration of propofol $1.5 \mu \mathrm{g} / \mathrm{ml}$, mean $\mathrm{BIS}=72$ ). However, some participants were responsive, and some were unresponsive to verbal commands during the ERP-recordings. This heterogeneity was due to the investigation of auditory processing under the a priori defined plasma concentration of $1.5 \mu \mathrm{g} / \mathrm{ml}$ propofol: both the level of awareness, and the BIS values under this concentration differ between individuals. Therefore, it is possible that the elicitation of MMN and ERAN was mainly due to the subgroup of less sedated participants, that is due to those subjects who were immediately responsive after ERP recordings under $1.5 \mu \mathrm{g} / \mathrm{ml}$ propofol.

It is, thus, not yet clear how MMN and ERAN are affected by deep propofol sedation in subjects unresponsive to verbal commands. To examine these issues, the present study investigated a homogeneous group with respect to the level of sedation (depth of anesthesia). For the clinician it is important to know which auditory mechanisms operate under this level of sedation because these mechanisms are a prerequisite for auditory awareness, auditory learning and

Table 1

The different levels of the modified observer's assessment of alertness/ sedation scale (MOAAS)

\begin{tabular}{ll}
\hline MOAAS Level & Response \\
\hline 5 & Responds readily to name spoken in normal tone \\
4 & Lethargic response to name spoken in normal tone \\
3 & Responds only after name is called loudly and/or \\
& repeatedly \\
2 & Responds only after mild prodding or shaking \\
1 & Responds only after painful stimuli \\
0 & No response after painful stimuli \\
\hline
\end{tabular}

In the present study, ERPs were collected under MOAAS level 5 (awake state and recovery period), and MOASS level 2-3 (deep sedation). 
memory, as well as language perception during anesthesia (Ghoneim, 2000).

Additionally, the present study investigated the MMN during recovery from anesthesia. Although some studies investigated the behavior of midlatency auditory evoked potentials during recovery from anesthesia (e.g. Rundshagen et al., 2002a,b), no published articles are available for the recovery of later ERPs (such as MMN, ERAN, and P3) from propofol anesthesia (for a study investigating the P3 under drug-induced amnesia and sedation see Veselis et al., 2001). Information about the recovery of these ERPs is potentially relevant, e.g. for the question of how to measure full cognitive recovery after anesthesia.

To shed light on these questions, the present study aimed at investigating if the neural mechanisms underlying the generation of both MMN and ERAN are still active in deeply sedated subjects (MOASS levels 2-3, with BIS values just above the reported values for adequate anesthesia). After the deep sedation, subjects were adequately anesthetized, and the MMN was measured during recovery from the propofol-induced unconsciousness to investigate if the MMN can be elicited immediately after subjects regain consciousness after propofol-induced anesthesia. It was hypothesized that both MMN and ERAN would be elicited during wakefulness (without any application of sedative drugs), but not during deep sedation and recovery phase.

\section{Materials and methods}

\subsection{Subjects}

Nineteen healthy male volunteers (20-31 years of age, mean age 24.9) participated in the study. Exclusion criteria included a history of deafness, obesity, hiatus hernia, or psychiatric disorder. All subjects were non-musicians who had not participated in any extracurricular music lessons or performances. The study was approved by the local ethics committee of the Medical Faculty, University of Leipzig, Leipzig, Germany. Written informed consent was obtained from each subject on the day prior to the measurement.

\subsection{Stimuli}

Two different stimulus types were used: an auditory oddball paradigm suited to elicit an MMN (MMN blocks), and a chord sequence paradigm suited to elicit an ERAN (ERAN blocks).

\subsubsection{MMN blocks}

Standard stimuli were piano-tones (General Midi sound no. 2) of $440 \mathrm{~Hz}$, presentation time was $600 \mathrm{~ms}$ (no pause between stimuli). Standards occurred with a probability of $80 \%$, and were intermixed equiprobably with 3 types of deviants: (a) frequency deviants, i.e. tones played with standard timbre but with deviant frequency $(495 \mathrm{~Hz}$, one tone above the standard tone), or (b) timbre deviants, i.e. tones played with standard frequency but deviant timbre (instrumental timbres other than piano, General MIDI sounds nos 4-20), or (c) omission deviants. Thus, each deviant (frequency-, timbre-, and omission deviant) occurred with a probability of $6.7 \%$. The order of presentation of deviants was pseudorandom, hence the type of deviant was unpredictable. Each deviant was preceded by at least 3 , but never more than 5 standards. The present study will only report effects of frequency- and timbre deviants, effects of omission deviants will be reported elsewhere (due to the length of this article).

\subsubsection{ERAN blocks}

The musical stimulus was identical, or similar to previous studies (Heinke et al., 2004b; Koelsch and Friederici, 2003). There were two sequence types, A and B (Fig. 1(a) and (b)). Both sequence types consisted of 5 chords. The first 4 chord functions were regular in-key chord functions. The final chord function of type A was a regular chord (tonic), of type B a music-syntactically irregular chord (dominant to the dominant). Compared to the Neapolitan chords used as irregular chords in the study from Heinke et al. (2004b), the arrangement of chords in the present study has the advantage that irregular chords did not physically confound with musical syntax (as a consequence, dominant to the dominant chords also represented a smaller degree of musical irregularity, e.g. because a dominant to the dominant is harmonically more closely related to the tonal center than a Neapolitan).

Each sequence type (regular, irregular ending) occurred with a probability of 0.5 , both sequence types were randomly intermixed. Each sequence was presented randomly in a tonal key different from the key of the preceding sequence (Fig. 1(c)). Most of the chords were
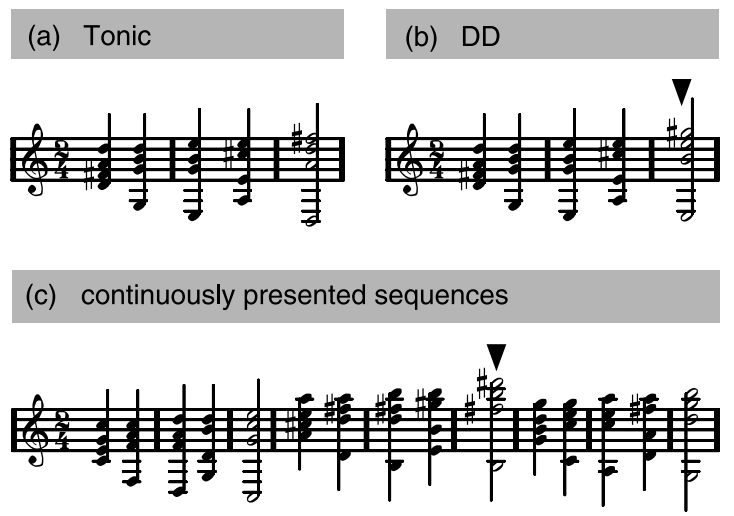

Fig. 1. Examples of musical stimuli. Chord sequences (in D-major), ending either on a regular tonic chord (a), or on a music-syntactically irregular dominant to the dominant (b). (c) In the experiment, sequences from all 12 major keys were presented in direct succession in pseudo-random order. Each sequence was presented in a tonal key that differed from the key of the preceding sequence, regular and irregular sequence endings occurred equiprobably. Arrows indicate irregular sequence endings. 
presented with a piano-sound (General Midi sound no. 2), presentation time of chords $1-4$ was $600 \mathrm{~ms}$ and chord 5 was presented for $1200 \mathrm{~ms}$. All chords were played in direct succession, there was no silence interval between chords or chord sequences. In $15 \%$ of the chord sequences, one chord was played with an instrumental timbre other than piano (similar to the MMN blocks).

All piano tones (and chords, respectively), had the same decay of loudness, like the normal decay of piano tones played at a rate of $6 / 10 \mathrm{~Hz}$. Stimuli were generated on a synthesizer (ROLAND JV 8010; Roland Corporation, Hamamatsu, Japan), and presented via headphones under computerized control.

Tone and chord-sequences were presented in sub-blocks, these sub-blocks were intermixed during each level. Each MMN sub-block comprised 30 frequency deviants, 30 timbre deviants, and 30 omission deviants. Each ERAN subblock comprised 90 sequences (including 13 sequences with a timbre deviant).

During the awake state, $3 \mathrm{MMN}$ - and 3 ERAN blocks were presented (resulting in a duration of $30 \mathrm{~min}$ ); under deep sedation, $9 \mathrm{MMN}$-and 9 ERAN blocks were presented (resulting in a duration of $90 \mathrm{~min}$ ). During recovery, 6 MMN blocks were presented (resulting in a duration of $30 \mathrm{~min}$ ); that is, no ERAN was measured during the recovery period (the investigation of the recovery period focused on the MMN to gain as high a temporal resolution as possible of the recovery of the MMN).

\subsection{Procedure}

Subjects were instructed to relax and to keep their eyes closed during the entire experiment. They were informed about the different blocks (MMN and ERAN), instructed to detect the tones and chords played with a deviant instrumental timbre, and to indicate their detection by pressing a response button. Examples of each stimulus type (tone- and chord sequences with and without timbre deviants) were presented before starting the measurements.
Before measurements of the recovery period, participants were asked if they still remembered the task (all participants reported that they were still aware of the task). Instead of employing a passive listening paradigm, the use of a task was imperative in the present study to control the behaviour of subjects during the deep sedation phase: for this phase, it was necessary to evaluate only those trials during which participants were actually deeply sedated. However, some subjects also had short passages in which they were less deeply sedated (sedation was then immediately increased, and respective EEG epochs were excluded from further analysis, see Sections 2.4 and 2.5 below). The level of sedation was assessed by obtaining the MOAAS and BIS values (see also below). In addition to these measures, the timbre detection task provided the necessary reassurance that subjects were appropriately sedated.

\subsection{Anesthesia}

Subjects were prepared for anesthesia after they had been instructed about the procedure of the experiment. An intravenous catheter was placed into a right forearm vein for the administration of propofol. Propofol is one of the most commonly used intravenous anesthetics. Due to its excellent pharmacokinetic (short half-life time) and pharmacodynamic properties (little side effects such as post-operative nausea and vomiting) the drug is particular suited for ambulatory surgery. The electrocardiogram and arterial oxygen saturation were continuously monitored during the entire experiment and non-invasive blood pressure was recorded every $5 \mathrm{~min}$. Participants breathed air throughout the study. If necessary, upper airway obstruction was relieved by gentle skin support.

EEG-measurements were performed during 3 different states of consciousness (Fig. 2): (a) wakefulness (this level served as a reference condition), (b) deep propofol sedation, and (c) recovery from propofol-induced unconsciousness. Propofol was administered by means of target-controlled infusion (Disoprifusor ${ }^{\circledR}$, Becton Dickinson Infusion

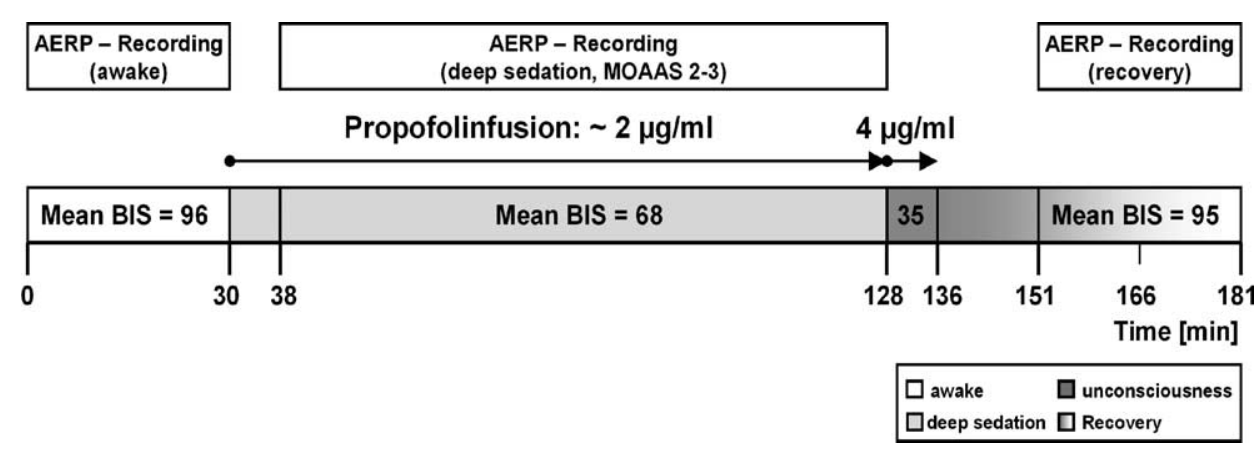

Fig. 2. Experimental procedure (for details see Section 2). First, subjects were measured for 30 min during wakefulness (i.e. without administration of propofol). Then, propofol-infusion was commenced to achieve the targeted level of sedation (MOAAS 2-3); this level was achieved in all subjects after about $8 \mathrm{~min}$. Then, subjects were measured with EEG over a period of $90 \mathrm{~min}$ (during this period, the level of sedation was kept constant by adapting the propofol infusion). After this period, the propofol-infusion was increased from 2 to $4 \mu \mathrm{g} / \mathrm{ml}$ to achieve unconsciousness. Again, this period had a duration of about $8 \mathrm{~min}$. Then, the propofol-infusion was stopped (i.e. subjects began to recover from anesthesia). Fifteen minutes later, ERPs were recorded for a duration of $30 \mathrm{~min}$ (while subjects recovered, and returned to normal BIS-values). 
Systems, Brezins, France). The Modified Observer's Assessment of Alertness and Sedation Scale (MOAAS, Chernik et al., 1990, see Table 1) was used to evaluate the level of sedation. Each experimental session started with EEG-recordings during wakefulness (MOAAS level 5). Then, the target for the plasma propofol concentration was set at $2 \mu \mathrm{g} / \mathrm{ml}$ to induce a MOAAS level 2-3. EEG recording was not started before this level was achieved. Sedation was kept on a constant MOAAS level by adapting the target-plasma concentration of propofol by the second author. The MOAAS level was assessed every $3 \mathrm{~min}$. In addition, the effect of propofol was evaluated online using the bispectral index (A-2000 ${ }^{\mathrm{TM}}$ BIS monitor, system version 2.10, BIS-algorithm 3.4, Aspect Medical Systems, Natick, MA, USA). This procedure guaranteed that ERPs were recorded at a stable level of sedation. As values of MOAAS levels, BIS values were recorded for subsequent statistical analysis every $3 \mathrm{~min}$ after starting the experiment.

After the presentation of the stimuli during sedation, propofol anesthesia was increased by setting the target plasma concentration at $4 \mu \mathrm{g} / \mathrm{ml}$. This plasma concentration was targeted over a period of $8 \mathrm{~min}$ because this time is required to achieve the desired effect-site concentration of propofol (i.e. the concentration of propofol in the brain) which reflects the actual actions of the drug (Billard et al., 1997). An effect-site concentration of propofol of $4 \mu \mathrm{g} / \mathrm{ml}$ was regarded as high enough to produce unconsciousness in all subjects (this was reflected in the BIS, Johansen and Sebel, 2000, see also Section 3). As soon as adequate anesthesia was established, propofol infusion was stopped and subjects recovered from anesthesia. Fifteen minutes after termination of the propofol administration, EEGs were recorded again over a period of $30 \mathrm{~min}$ to investigate the recovery of ERPs from anesthesia.

Additionally, venous blood samples were obtained at the beginning of the EEG-measurements during recovery (i.e. 15 min after cessation of the propofol infusion), after the first half of the EEG-measurements during recovery (i.e. 30 min after stopping the propofol infusion) and at the end of EEG-measurements (i.e. $45 \mathrm{~min}$ after stopping the propofol infusion) to determine the plasma concentration of propofol during the recovery period. Each blood sample for determination of the propofol plasma level was collected in a heparinized tube, and centrifuged at $2800 \mathrm{rpm}$ for 15 min. Plasma propofol concentrations were measured by high-pressure liquid chromatography.

\subsection{EEG-recording and data analysis}

Electroencephalographic (EEG) data were measured with $\mathrm{Ag}-\mathrm{AgCl}$ Electrodes, digitized with a sampling rate of $500 \mathrm{~Hz}$ using the following 18 electrode positions of the international 10-20 system: Fz, F3, F4, F7, F8, FT7, FT8, FC3, FC4, Cz, C3, C4, T7, T8, CP5, CP6, as well as left (M1) and right (M2) mastoid sites. Because participants were lying in supine position during the experiment, no electrodes were placed over parietal and occipital areas. Note, that the measured electrode sites are sufficient for a valid identification of MMN (Schröger, 1998) and ERAN (Koelsch and Friederici, 2003). An electrode placed on the nose served as reference, the ground-electrode was located on the chest. To control for artifacts caused by eye movements, vertical and horizontal electro-oculograms were recorded bipolarly.

After the measurements, raw data were filtered using a $0.25 \mathrm{~Hz}$ highpass filter (1001 points, finite impulse response). For artifact rejection, each sampling point was centered in a gliding window, and rejected if the standard deviation exceeded $50 \mu \mathrm{V}$ either within a $200 \mathrm{~ms}$, or an $800 \mathrm{~ms}$ gliding window (this procedure was applied for each channel, as well as for vertical and horizontal EOGs). The two standards following directly a deviant in the MMN blocks were excluded from further evaluation. For every subject, artefact-free EEGepochs lasting from -50 to $1200 \mathrm{~ms}$ with respect to stimulus onset were averaged off-line using a $50 \mathrm{~ms}$ pre-stimulus baseline. Then, grand-averages were calculated for each condition across all subjects.

For statistical analyses of ERP effects, EEG data were re-referenced off-line to the algebraically mean of both mastoid electrodes. Mean amplitude values of ERPs were computed for electrode regions of interest: left frontocentral (C3, F3, FC3), right fronto-central (C4, F4, FC4) and centro-parietal (CP5, CP6). Time windows used for analyses were centered around the maxima of effects.

ERPs were analyzed statistically by repeated-measures ANOVAs as univariate tests of hypotheses for within subject effects. If not separately indicated, the following ANOVAs were conducted for the MMN blocks (results are reported in Table 2): ERPs with frontal, or fronto-central scalp distribution were tested by computing ANOVAs for the two frontocentral ROIs separately for each level (awake state, deep sedation, recovery) with factors deviance (two levels: standard, deviant), and hemisphere. P3b potentials were tested separately for each level by computing ANOVAs for the centro-parietal ROI (CP5, CP6) with factor deviance. Three standard ANOVAs are reported for each level: (a) ANOVAs for standards vs. frequency deviants, (b) ANOVAs for standards vs. timbre deviants, and (c) ANOVAs for frequency deviants vs. timbre deviants. In addition, ANOVAs for the pooled deviants (i.e. standards vs. pooled frequency and timbre deviants) are reported for the deep sedation level. The following ERPs were tested (with time windows used for computation of ANOVAs in parentheses): MMN (100-130 ms), N2b (200$230 \mathrm{~ms}$ ), P3a (awake state and recovery: $280-350 \mathrm{~ms}$; deep sedation: $220-280 \mathrm{~ms}$ ), P3b (awake state and deep sedation: 280-350 ms; recovery: $320-400 \mathrm{~ms}$ ), late negativity (450$600 \mathrm{~ms})$. To compare ERP amplitudes between levels, ANOVAs were conducted with factors deviance, and level of sedation (see Section 3 for details).

Analogously, for the data obtained in the ERAN blocks, ANOVAs were carried out separately for levels awake state 
Table 2

Summary of ANOVAs for the MMN blocks

\begin{tabular}{|c|c|c|c|c|c|}
\hline & \multicolumn{5}{|l|}{$F_{1,18}$ and $P$-values } \\
\hline & $\overline{\mathrm{MMN}}(100-130 \mathrm{~ms})$ & $\mathrm{N} 2 \mathrm{~b}(200-300 \mathrm{~ms})$ & P3a $(280-350 \mathrm{~ms})$ & P3b $(280-350 \mathrm{~ms})^{\mathrm{a}}$ & Late neg. $(450-600 \mathrm{~ms})$ \\
\hline \multicolumn{6}{|l|}{ Awake state } \\
\hline Frequency & $39.04,0.0001$ & n.s. & $34.36,0.0001$ & n.s. & $10.09,0.006$ \\
\hline Timbre & $48.81,0.0001$ & $5.17,0.05$ & $5.18,0.05$ & $23.19,0.0001$ & $20.81,0.0002^{\mathrm{b}}$ \\
\hline Frequency vs. timbre & $8.52,0.001$ & $7.61,0.02$ & n.s. & $5.64,0.05$ & $17.16,0.001$ \\
\hline \multicolumn{6}{|l|}{ Deep sedation } \\
\hline Frequency & $4.46,0.05$ & n.s. & $4.52,0.05^{\mathrm{c}}$ & n.s. & $6.54,0.02$ \\
\hline Timbre & $3.25,0.09$ & n.s. & $11.07,0.005^{\mathrm{c}}$ & n.s. & $10.06,0.006$ \\
\hline Pooled deviants & $5.57,0.05$ & n.s. & $11.73,0.005^{\mathrm{c}}$ & n.s. & $9.29,0.007$ \\
\hline Frequency vs. timbre & n.s. & n.s. & n.s. ${ }^{\mathrm{c}}$ & n.s. & n.s. \\
\hline \multicolumn{6}{|l|}{ Recovery } \\
\hline Frequency & $85.93,0.0001$ & n.s. & n.s. & n.s. ${ }^{d}$ & $25.38,0.0001$ \\
\hline Timbre & $120.21,0.0001$ & $5.35,0.05$ & n.s. & n.s. ${ }^{d}$ & $40.05,0.0001$ \\
\hline Frequency vs. timbre & $45.87,0.0001$ & $7.84,0.02$ & n.s. & n.s. ${ }^{d}$ & $22.37,0.0002$ \\
\hline
\end{tabular}

ANOVAs were conducted with factors deviance (standard, deviant tones) and hemisphere for fronto-central ROIs (except for P3b-ANOVAs, which were carried out for the centro-parietal ROI, and with factor deviance only).

${ }^{\text {a }}$ Parietal ROI.

${ }^{\mathrm{b}}$ Interaction deviance $\times$ hemisphere: $P<0.05$.

c Time-window 220-280 ms.

d Time-window 320-400 ms.

and deep sedation (as mentioned above, no ERAN block was employed during recovery). ANOVAs were computed for the fronto-central ROIs with factors deviance (two levels: regular, irregular chord sequence endings), and hemisphere (results are reported in Table 3). The following ERPs were tested (with time windows used for computation of ANOVAs in parentheses): ERAN (160-230 ms), and late negativity (450-600 ms).

To guarantee that ERPs calculated for the level of deep sedation were only recorded during phases in which subjects had a level of sedation corresponding to MOAAS 2-3, all sequences in which participants responded behaviorally to the deviant instruments were excluded from further data analysis; moreover, if a subject responded to a deviant instrument, all sequences preceding the deviant instrument (up to the last occurrence of a deviant instrument to which the subject did not respond to), as well as all following sequences (up to the next deviant instrument to which the subject did not respond to) were excluded.

For the awake state, as well as for the recovery period, only ERPs recorded during phases in which the subjects responded to the timbre deviants were analyzed (i.e. all sequences during which participants did not respond to timbre deviants were excluded from further data analysis).

\section{Results}

\subsection{Awake state}

Behaviorally, subjects detected on average $92.4 \%$ of the timbre deviants $(\mathrm{SD}=8.2$, range $64-100)$. Hit rates did not significantly differ between MMN blocks (90.8\%) and
ERAN blocks $(95.5 \%)$. On average there were $0.2 \%$ false alarms ( $\mathrm{SD}=0.2$, range $0.0-0.8)$.

In the MMN blocks, both frequency and timbre deviants elicited an MMN that was frontally maximal and inverted polarity at mastoid electrodes (Fig. 3(a), see Table 2 for ANOVAs). Both deviants elicited a distinct P3a (peaking at frontal electrodes at around $320 \mathrm{~ms}$ ), and a late frontal negativity. The timbre MMN was followed by an $\mathrm{N} 2 \mathrm{~b}$ (maximal fronto-centrally at around $200 \mathrm{~ms}$ ), and by a P3b that was maximal at around $350 \mathrm{~ms}$ over the centro-parietal sites (CP5, CP6). It appears that the frequency deviants also elicited small $\mathrm{N} 2 \mathrm{~b}$ and $\mathrm{P} 3 \mathrm{~b}$ potentials, which both overlapped with the P3a. Statistically, all effects, except P3a, had a larger amplitude when elicited by the timbredeviants (Table 2).

In the ERAN blocks, irregular chord sequence endings elicited an ERAN, which was followed by a small late negativity, both potentials were not lateralized, had frontal predominance and inverted polarity at mastoidal sites (as expected; see Fig. 4(a) and Table 3 for ANOVAs). The ERAN had a smaller amplitude than the frequency (and the timbre) MMN: an ANOVA comparing the amplitudes of ERAN (160$230 \mathrm{~ms})$ and frequency-MMN (100-130 ms) with factors

Table 3

Summary of ANOVAs for the ERAN blocks (fronto-central ROIs)

\begin{tabular}{lll}
\hline & $F_{1,18}$ and $P$-values & \\
\cline { 2 - 3 } & ERAN $(160-230 \mathrm{~ms})$ & Late neg. $(450-600 \mathrm{~ms})$ \\
\hline Awake state & $39.71,0.0001$ & $7.90,0.02$ \\
Deep sedation & n.s. & n.s. \\
\hline
\end{tabular}

ANOVAs were conducted with factors deviance (regular, irregular chords) and hemisphere. 


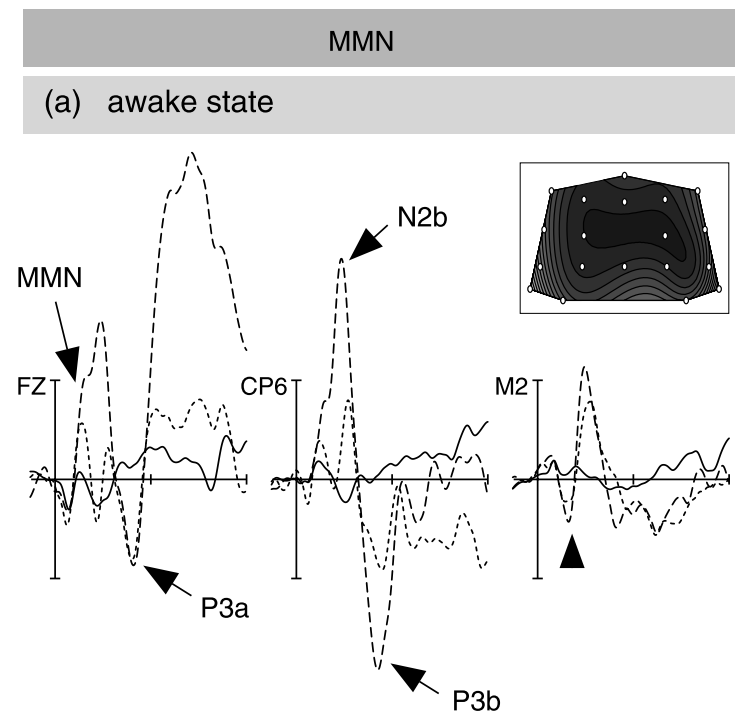

(b) deep sedation

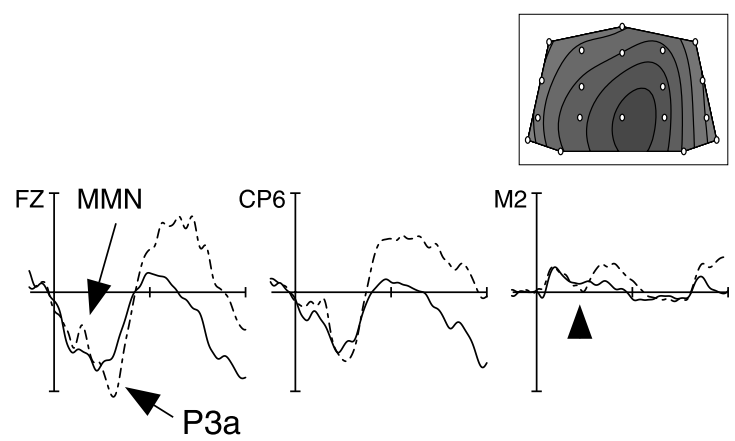

(c) recovery
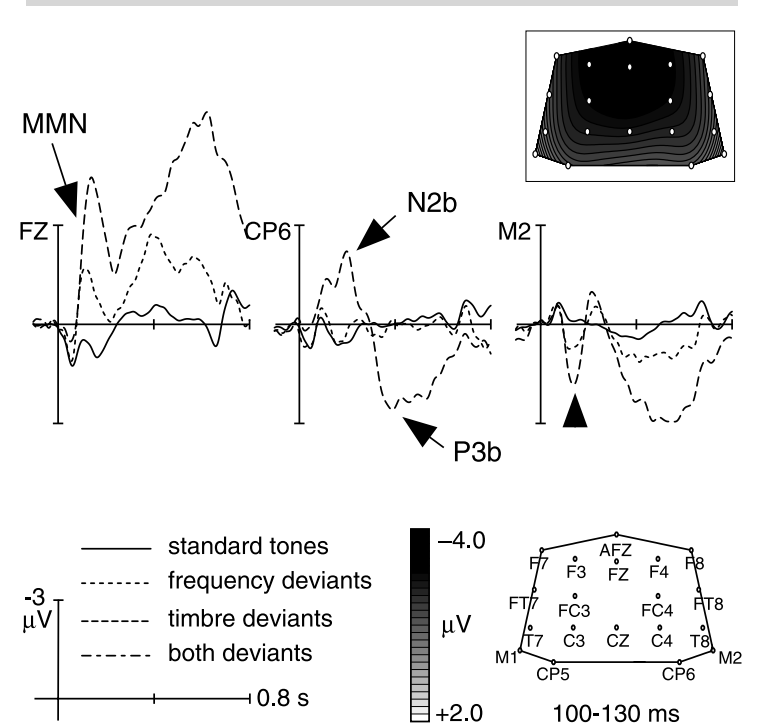

Fig. 3. ERPs elicited in the MMN blocks during the awake state (a), during deep sedation (b), and during the recovery period (c). The insets show the scalp distribution maps of the MMN (difference potentials: standards subtracted from both deviants, view from top, nose is upwards), interpolated over the time window used for statistical analysis (100$130 \mathrm{~ms}$ ). The ERP waveforms show that both MMN and P3a (as well as a late negativity) were elicited under deep sedation. The amplitude of the

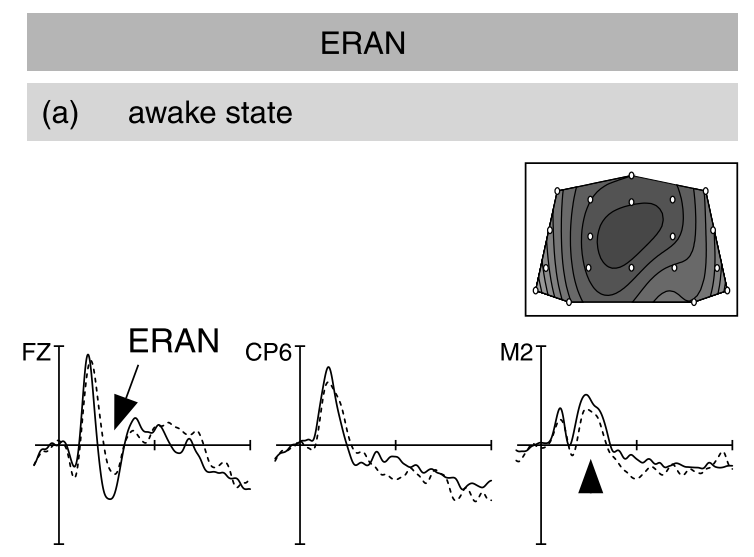

(b) deep sedation

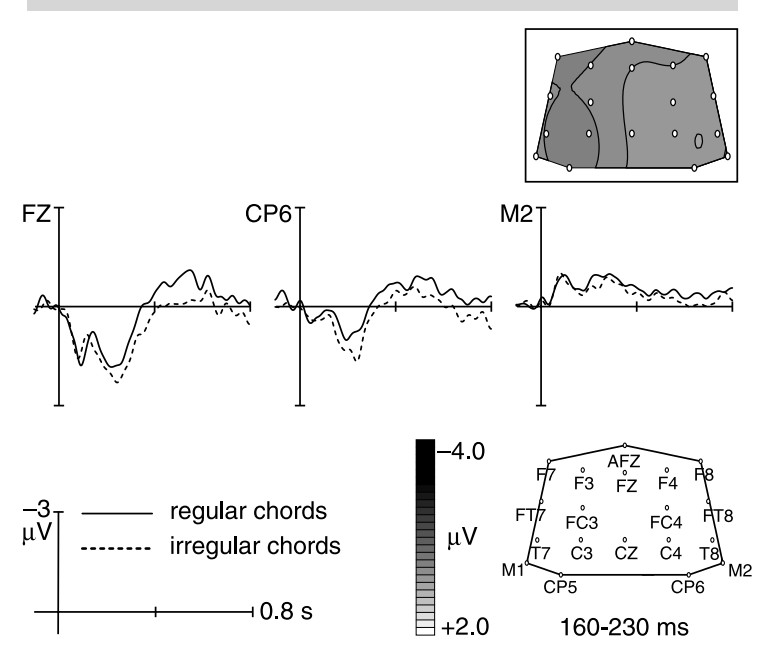

Fig. 4. ERPs elicited in the ERAN blocks during the awake state (a), and during deep sedation (b). The insets show the scalp distribution map of the ERAN (difference potentials: regular subtracted from irregular chords, view from top, nose is upwards), interpolated over the time window used for statistical analysis (160-230 ms). During the awake state, a clear ERAN was elicited (followed by a late negativity; the polarity inversion of the ERAN at mastoid leads is indicated by the arrow head). No ERAN was present in the ERPs measured during deep sedation.

stimulus type (tones, chords), and deviance (standard, deviant) indicated an effect of stimulus type $(F(1,18)=24.50, P<$ $0.0001)$, an effect of deviance $(F(1,18)=52.86, P<0.0001)$, and a two-way interaction $(F(1,18)=5.14, P<0.05)$.

\subsection{Deep sedation}

Behaviorally, participants responded to $9.6 \%$ of the timbre deviants $(\mathrm{SD}=7.3$, range 1.6-28.3). Hit rates did not

MMN elicited during the recovery period virtually did not differ from the MMN elicited during the awake state. In all levels, the MMN inverted polarity at mastoid leads (arrow heads). Note that no P3a was observed during the recovery period, and that the P3b is markedly reduced during recovery (compared to the awake state). 
differ statistically between MMN blocks (10.4\%) and ERAN blocks (8.2\%).

As described in Section 2, epochs in which participants were not deeply sedated (according to MOAAS 2-3) were excluded from further data analysis (so that the ERPs presented below did not contain any trials in which participants responded behaviorally to the timbre deviants). In the MMN blocks, data from both frequency and timbre deviants were pooled to increase the signal-to-noise-ratio (SNR), because potentials elicited by both deviants virtually did not differ from each other (ANOVAs did not indicate a difference between frequency- and timbre-ERPs for any of the time-windows listed in Table 2). In the waveform of the pooled deviants, a significant MMN was present, although the MMN amplitude was considerably smaller compared to the awake state (Figs. 3(b) and 5, see Table 2 for ANOVAs). The MMN peaked around $120 \mathrm{~ms}$ and inverted polarity at mastoid leads.

The MMN in the pooled data was not simply due to refractoriness-related effects of the $\mathrm{N} 1$ of the timbre deviants, as indicated by a significant MMN elicited by the frequency deviants only (Table 2): the effect elicited by the frequency-deviants is mainly an MMN because the frequency difference between standards and frequency deviants was only around 12\% (larger differences in frequency between standards and deviants may lead to differences in $\mathrm{N} 1$ amplitudes that are related to refractoriness effects, rather than to sensory memory operations, see, e.g. Schröger, 1998).

To test the amplitude difference of the frequency MMN between awake state and deep sedation, an ANOVA with factors level (awake state, deep sedation) and deviance was computed; results indicated main effects for both factors $(P<0.0001$ in each test $)$, and a two-way interaction $(F(1,18)=7.66, P<0.05$ tested one-sided $)$. The analogous

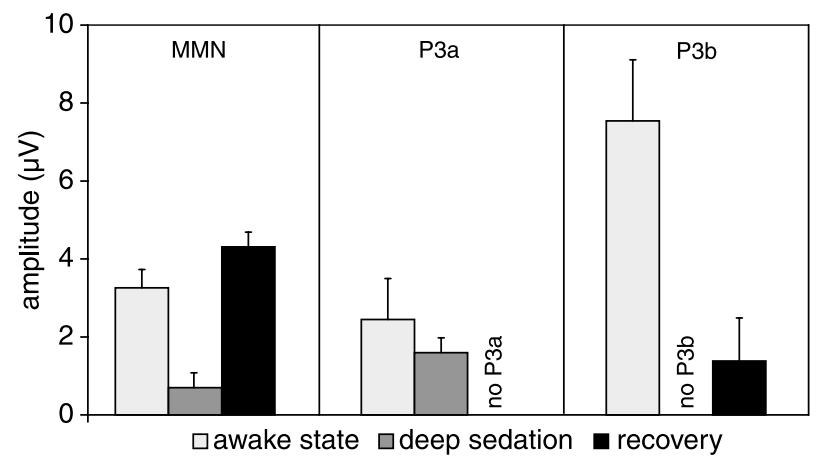

Fig. 5. Mean amplitudes (and SDs) of MMN (pooled deviants), P3a, and P3b, separately for awake state, deep sedation, and recovery. Mean amplitudes were calculated from difference potentials (standards subtracted from deviants) for the time-windows and electrodes used for statistical analyses (see Section 2). Compared to the awake state, the MMN amplitude was markedly reduced during deep sedation, but not during the recovery period. Nevertheless, a clear P3a was elicited under deep sedation, but not during recovery. The amplitude of the $\mathrm{P} 3 \mathrm{~b}$ was strongly reduced (and statistically not significant) during the recovery period.
ANOVA for the pooled deviants (frequency and timbre MMN) also revealed a two-way interaction $(F(1,18)=$ 34.80, $P<0.0001)$.

To guarantee that the amplitude reduction of the MMN is not simply due to the absence of $\mathrm{N} 2 \mathrm{~b}$ potentials during deep sedation, an ANOVA was also carried out for the data recorded at the mastoid electrodes (the N2b does not invert polarity at mastoid leads, data were referenced to the nose electrode for this analysis). The ANOVA was computed for the pooled deviants with factors level and deviance. Results indicated main effects for both factors $(P<0.01$ in each test), and a two-way interaction $(F(1,18)=11.79, P<$ $0.005)$.

No N2b or P3b was elicited, neither by timbre-, nor by frequency deviants (unlike to the awake state), reflecting that participants did not behaviorally respond to the deviants included in the data analysis. However, both deviants elicited a clear P3a (being maximal at frontal leads at around $250 \mathrm{~ms}$ ), and a clear late negativity (Fig. 3(b) and Table 2). The P3a peaked slightly earlier than in the awake state, presumably because the $\mathrm{P} 3$ a elicited during the awake state was partly overlapped by the $\mathrm{N} 2 \mathrm{~b}$.

In the music condition, no ERAN was elicited by the irregular sequence endings (Fig. 4(b) and Table 3; nominally, the ERP values of irregular chords were even slightly more positive than those of regular chords). To compare the ERAN elicited during the awake state with the ERPs elicited during deep sedation, an ANOVA was conducted with factors level, and deviance (regular, irregular chords) for a time-interval from 160 to $230 \mathrm{~ms}$. This ANOVA indicated an effect of deviance $(P<0.0005$, this effect is due to the clear ERAN elicited during awake state), and an interaction between factors level and deviance $(F(1,18)=22.98, P<$ 0.0001; no main effect of level was indicated, presumably because the P2 had a very similar amplitude in both levels, see also Fig. 4).

\subsection{Recovery}

After the sedation phase, propofol anesthesia was increased (target plasma concentration $=4 \mu \mathrm{g} / \mathrm{ml}$ ) over a period of 8 min to produce unconsciousness in all subjects (reflecting adequate anesthesia, mean BIS-value was 35, $\mathrm{SD}=5$; see also Table 4). Within the $15 \mathrm{~min}$ after cessation of the propofol infusion (i.e. before starting the measurements), all subjects regained consciousness. On average, subjects responded to verbal commands spoken with normal loudness for the first time $10.9 \mathrm{~min}(\mathrm{SD}=3$, range 5-15) after stopping the propofol infusion. The corresponding average BIS value at this time was $63.4(\mathrm{SD}=15$, range 3289). The mean BIS value during the first half of the recovery period was $94(\mathrm{SD}=5)$, and $95(\mathrm{SD}=3)$ during the second half (Table 4).

Means of propofol plasma levels during the recovery period were $1.62 \mu \mathrm{g} / \mathrm{ml}(\mathrm{SD}=0.6$, range $2.88-0.88)$ at the beginning of the EEG-measurements, $0.96 \mu \mathrm{g} / \mathrm{ml}(\mathrm{SD}=0.3$, 
Table 4

Physiological parameters measured during the different levels of consciousness: systolic, diastolic, and mean blood pressure (Sys BP, Dias BP, and Mean BP) in $\mathrm{mmHg}$, heart rate $(\mathrm{HR})$ in beats per minute, arterial oxygen saturation $\left(\mathrm{SpO}_{2}\right)$ in percent, and bispectral index (BIS)

\begin{tabular}{|c|c|c|c|c|c|c|}
\hline & Sys BP & Dias BP & Mean BP & HR & $\mathrm{SpO}_{2}$ & BIS \\
\hline Awake state & $119(9)$ & $69(5)$ & $86(5)$ & $64(8)$ & $98(1)$ & $96(2)$ \\
\hline Deep sedation & $106(9)^{*}$ & $59(6)^{*}$ & $75(6)^{*}$ & $64(8)$ & $96(1)^{*}$ & $68(4)^{*}$ \\
\hline Unconsciousness & $102(9)^{*}$ & $56(7)^{*}$ & $70(6)^{*}$ & $64(9)$ & $94(2)^{*}$ & $35(5)^{*}$ \\
\hline Recovery (1) & $110(8)^{*}$ & $64(8)^{*}$ & $79(7)^{*}$ & $62(11)$ & $97(2)$ & $94(5)^{*}$ \\
\hline Recovery (2) & $111(7)$ & $67(8)$ & $81(7)$ & $60(9)$ & $98(1)$ & $95(3)$ \\
\hline
\end{tabular}

Significant changes of values (compared to those of the directly preceding level) are marked by asterisks $(P<0.05$, two-tailed $t$ tests).

range 1.86-0.64) after the first half of the recovery measurements, and $0.78 \mu \mathrm{g} / \mathrm{ml}(\mathrm{SD}=0.2$, range $1.38-$ 0.35 ) at the end of the recovery period. Two-tailed $t$ tests indicated that the differences in mean plasma concentrations were statistically significant (beginning vs. measurement after first half: $P<0.0001$; measurement after first vs. measurement after second half: $P<0.0001)$.

When asked before starting the measurements, all participants reported that they still remembered the task. Behaviorally, subjects detected on average $87.9 \%$ of the timbre deviants ( $\mathrm{SD}=14.9$, range 36.6-100). The difference in hit percentages between awake state and recovery was statistically significant $(P<0.05$, two-tailed $t$ test $)$. Although hit rates were nominally lower during the first half of the recovery period (first half: $86.3 \%$, second half: $89.6 \%$ ), this difference between halves was statistically not significant. On average there were $0.5 \%$ false alarms per subject $(\mathrm{SD}=0.6$, range $0.0-2.3)$. Mean reaction time was $662 \mathrm{~ms}(\mathrm{SD}=181)$ during the recovery. Reaction times were virtually identical compared to the awake state (awake state: $633 \mathrm{~ms}, \mathrm{SD}=125$; the slight difference in reaction times between levels was statistically not significant).

For the ERP analysis, the few epochs in which participants did not respond to the timbre deviants were excluded from further analysis (i.e. only epochs in which participants responded were included in the data analysis; see Section 2). This procedure was applied to the data of both the awake state and the recovery period, so that possible differences between ERPs of recovery and awake state (especially between those related to behavioral performance such as $\mathrm{N} 2 \mathrm{~b}$ and $\mathrm{P} 3 \mathrm{~b}$ ) cannot be due to a different percentage of epochs in which participants did not respond to the timbre deviants.

As in the awake state, both deviants elicited a clear MMN with frontal preponderance and polarity inversion at mastoid leads (Figs. 3(c) and 5, see Table 2 for ANOVAs). The amplitude of the frequency MMN was nominally even larger than the MMN elicited during the awake state, but this difference was statistically not significant. Even when examining the first half of the recovery only, a clear MMN was elicited by the frequency deviants (with an amplitude that was nominally larger than the amplitude of the MMN of the awake state, but again this difference was statistically not significant). The amplitude of the frequency-MMN did statistically not differ between the first and the second half of the recovery period: an ANOVA with factors halves (first half vs. second half of recovery period), and deviance indicated an effect of deviance $(F(1,18)=85.31, P<$ $0.0001)$, but no effect of half $(P>0.7)$, and no two-way interaction $(P>0.3)$.

Like the frequency deviants, the timbre deviants also elicited a clear MMN. Again, the timbre-MMN elicited during the recovery period was even larger than the MMN recorded during the awake state (an ANOVA with factors level and deviance revealed a two-way interaction, $P<$ 0.05), possibly due to the absence of a P3a.

Interestingly, in contrast to the two previous levels (awake state and deep sedation), no P3a was observed in the ERP waveforms of deviants. To compare the P3a amplitudes of both deviants (pooled data) between deep sedation and recovery, an ANOVA was conducted with factors level and deviance. Results indicated an effect of level $(F(1,18)=54.80, \quad P<0.0001)$, and a two-way interaction $(F(1,18)=9.32, P<0.007)$.

Moreover, no $\mathrm{N} 2 \mathrm{~b}$ was observed in response to the frequency deviants, and only a small $\mathrm{N} 2 \mathrm{~b}$ was elicited by the timbre deviants (compared to the awake state). Similarly, for both deviants the P3b potential was markedly reduced compared to the awake state (the P3b was visible only at the centro-parietal electrodes and statistically not significant for either of the two deviants, see Figs. 3(c) and 5, and Table 2 for ANOVAs). To compare the amplitude of the P3b elicited by the timbre deviants between awake state and recovery, an ANOVA was conducted for the parietal ROI with factors level (awake state, recovery) and deviance (standards, timbre deviants). Results indicated effects for both factors (level: $F(1,18)=27.38, P<0.0001$; deviance: $F(1,18)=$ 13.21, $P<0.002)$, and an interaction between the two factors $(F(1,18)=28.66, P<0.0001)$. Even if only the second half of the recovery period was examined, the P3b amplitude was clearly reduced compared to the awake state $(F(1,18)<25.89, P<0.0001)$.

That is, although a P3b is visible in the ERP waveforms, the P3b was statistically not significant. The following section examines if this missing significancy is due to a larger between-subjects variance of P3b amplitudes than in the awake level, or if the amplitude reduction of the $\mathrm{P} 3 \mathrm{~b}$ (compared to the awake level) is due to a greater within- or between-subjects jittering of P3 latencies elicited across trials during the recovery period 
(note that only response-trials were included in the data analysis, and that it is, thus, not possible that the P3b was reduced simply because of trials in which participants did not respond to the timbre deviants).

To investigate these issues, subjects were divided into 3 groups: (1) subjects with a clear, phasic P3b during the recovery phase $(n=9),(2)$ subjects with a tonic $\mathrm{P} 3 \mathrm{~b}(n=5)$, and (3) subjects with no P3b $(n=5$; in the awake level, all participants except one showed a clear, phasic P3b). Fig. 6(a) shows the reaction times and standard deviations of reaction times for all subjects, separately for the awake state and the recovery period. As can be seen in Fig. 6(a), the standard deviation of response times did not differ between the two levels, in none of the 3 subgroups. Standard deviations calculated for each of the 19 subjects, and each of the two levels (awake state, recovery), were compared between levels by a one-sample, two-tailed $t$ test; this test did not indicate a significant difference between the two levels $(P>0.1)$. Likewise, an ANOVA with factors level and group (clear P3b, tonic P3b, no P3b) did not indicate a

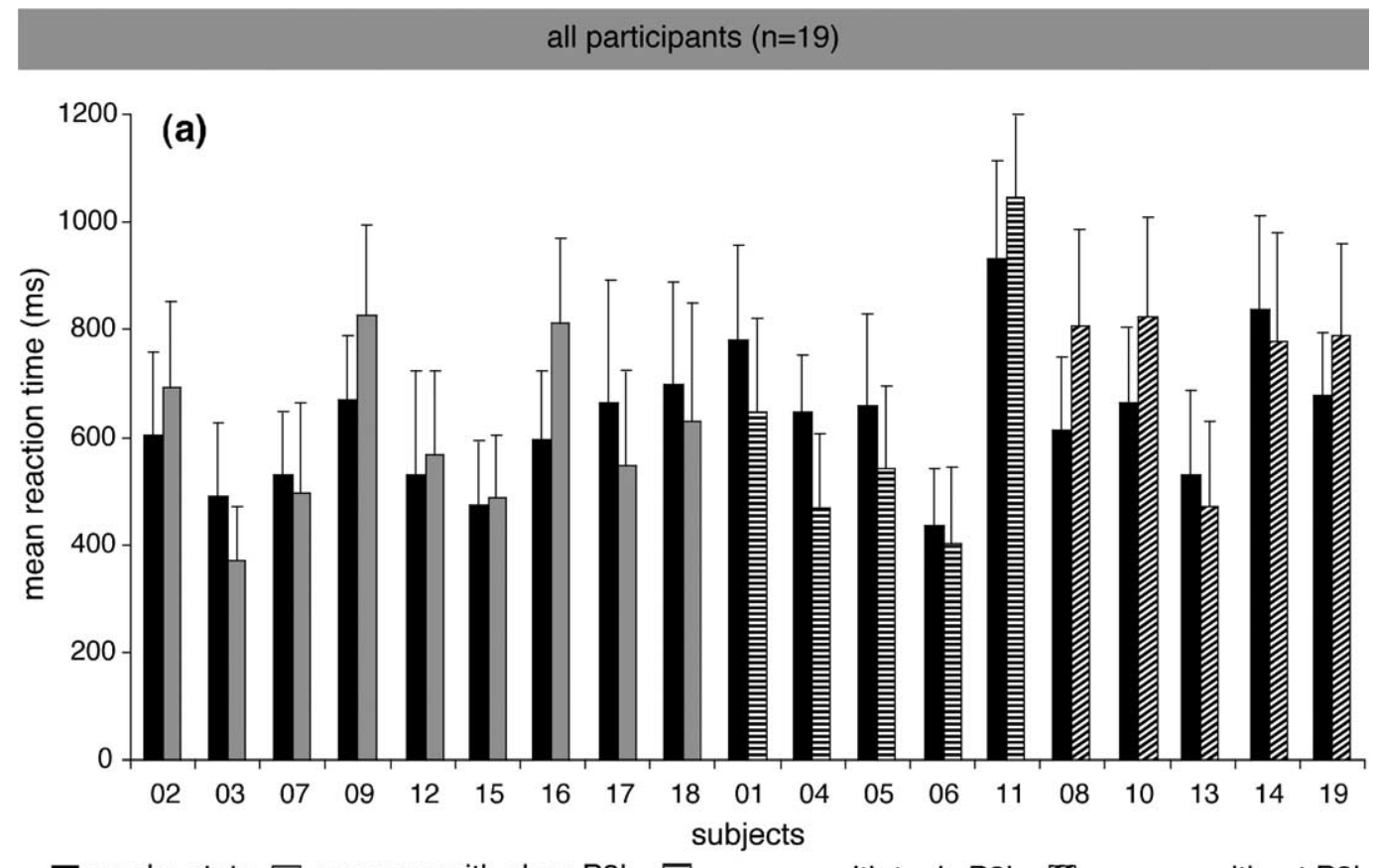

awake state $\square$ recovery with clear P3b

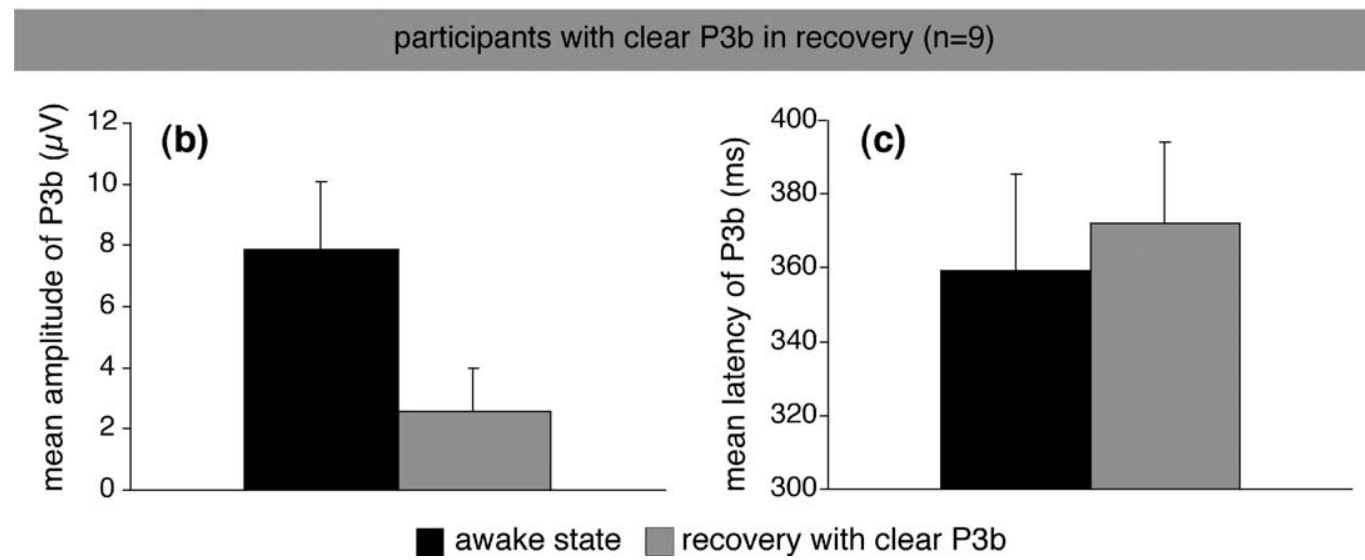

Fig. 6. (a) Means and SDs of response times, separately for each subject for the awake state (black columns), and for the recovery period. Behavioral data of the recovery period are differentiated between subjects who showed a clear P3b during the recovery period (grey columns), subjects with a tonic P3b (horizontally striped columns), and subjects with no clear P3b during recovery (diagonally striped columns). In all subgroups, the variance of reaction times virtually did not differ between levels. (b) Mean amplitudes and SDs of P3b potentials (difference potentials: standards subtracted from deviants) for subjects with clear P3b during the recovery period, separately for the awake state (black column), and the recovery period (grey column), calculated for the time-window and for the electrodes used for statistical analysis (320-400 ms, CP5 and CP6). The data indicate that even in subjects with a clear P3b, the P3b was markedly reduced during the recovery period. (c) Mean latencies and SDs of P3b potentials for subjects with clear P3b during the recovery period, separately for the awake state (black column), and the recovery period (grey column), calculated for the time-window and for the electrodes used for statistical analysis (320-400 ms, CP5 and CP6). P3b latencies were very similar in both levels, and the variance of P3b latencies was not greater during the recovery period, indicating that the amplitude reduction of the $\mathrm{P} 3 \mathrm{~b}$ was not due to a greater between-subjects jittering of P3b potentials. 
two-way interaction $(P>0.2$; additional Tukey HSD posthoc tests did not indicate any effect of level for any of the 3 groups). Because the $\mathrm{P} 3 \mathrm{~b}$ latency correlates with reaction time (Donchin, 1979), these findings suggest that the reduced P3b in subgroup 1, the tonic P3b in subgroup 2, and the absence of the P3b in subgroup 3, is not due to a greater jittering of $\mathrm{P} 3 \mathrm{~b}$ latencies elicited in each trial during the recovery period.

Fig. 6(b) shows that the P3b amplitude is markedly reduced even in subgroup 1 (subjects with clear, phasic $\mathrm{P} 3 \mathrm{~b}$ peak in the recovery period; $T(8)=7.6, P<0.0001$ ), indicating that the neural mechanisms underlying the generation of the $\mathrm{P} 3 \mathrm{~b}$ were impaired during the measured recovery period. The peak-latency of the P3b (as measured in subgroup 1) during the recovery period was similar to the P3b latency of the awake level (Fig. 6(c), the nominal difference between levels was statistically not significant), and the variance of latencies was nominally even slightly smaller (n.s.) during the recovery period. This makes it highly unlikely that the amplitude reduction in subgroup one is simply due to a greater between subjects jittering of $\mathrm{P} 3 \mathrm{~b}$ peak latencies.

In contrast to $\mathrm{P} 3 \mathrm{a}$ and $\mathrm{P} 3 \mathrm{~b}$, a late frontal negativity was present in the waveforms of both deviants, as in the previous levels (see also Table 2). The amplitude of this late negativity was larger during the recovery period than during deep sedation: an ANOVA for pooled deviants (frontocentral ROIs) for the time-interval from 450 to $600 \mathrm{~ms}$ with factors deviance and level (deep sedation, recovery) indicated an interaction between the two factors $(F(1,18)=4.97, P<0.05)$. Amplitudes of the late negativity did not statistically differ between recovery period and awake state $(P>0.5)$, even when analyzing timbre deviants only $(P>0.2)$.

\section{Discussion}

\subsection{Awake state}

During the awake state, an MMN was elicited by both timbre and frequency deviants. In contrast to the frequency deviants, the task-relevant timbre deviants elicited N2b, and P3b components (due to the detection of the target stimuli, Näätänen, 1992; Schröger, 1998). Both deviants elicited a clear P3a, and a late negativity. The P3a reflects attentionrelated cognitive mechanisms, and is taken to reflect an involuntary shift of attention due to the (potential) relevance of a perceived stimulus (Alho et al., 1997; Escera et al., 2000). Like the P3a, the late negativity presumably also reflects attention-related mechanisms. In the literature, this negativity was originally denoted as the reorienting negativity (RON), and taken to reflect reorienting of attention to a task-relevant dimension of an auditory stimulus after the automatic detection of a deviant in a task-irrelevant dimension (Schröger and Wolff, 1998).
However, the exact functional significance of this component is not yet clear.

The music-syntactically irregular chords elicited a clear ERAN, reflecting the violation of a musical sound expectancy, and the higher amount of syntactic analysis required by the irregular chord functions (Koelsch and Siebel, 2005). The ERAN was followed by a small late frontal negativity which has previously been denoted as N5, and taken to reflect processes of harmonic integration (Koelsch, 2005).

\subsection{Deep sedation}

Under deep propofol-induced sedation (MOAAS 2-3), i.e. when participants were unresponsive to normal verbal commands, a tiny MMN was observed in response to frequency and timbre deviants. The MMN was clearly reduced compared to the awake state. The presence of this MMN residual during deep sedation replicates findings from previous studies (Heinke et al., 2004b; Yppärilla et al., 2002), indicating that auditory sensory memory operations are markedly affected by sedation, but can still be observed under deep sedation, even when participants are unresponsive to normal verbal commands. Simpson et al. (2002) reported that a frequency-MMN was visible in ERPs recorded during deep sedation, but statistically not significant. The missing significancy in that study is presumably due to an insufficient number of trials, which did not produce a signal-to-noise ratio high enough to yield statistical significance (note that in the present study the duration of the deep sedation phase was $90 \mathrm{~min}$, resulting in the presentation of 300 deviants per subject in the MMN blocks).

Interestingly, a clear positivity with a latency of around $250 \mathrm{~ms}$ and frontal scalp distribution (usually denoted as P3a), as well as a clear late negativity were elicited under deep sedation. Notably, in our previous study which used an experimental paradigm similar to that of the present study (Heinke et al., 2004b), neither P3a nor late negativity were elicited under deep sedation, although the average BIS values were lower in the present study (that is, although subjects were on average even slightly deeper sedated in the present study than in the previous study). The difference between the two studies is that in the previous study subjects listened passively to the stimuli, whereas in the present study subjects were trained to respond to the timbre deviants during the awake state. That is, subjects had a task during the entire experiment, and they performed this task actively for $30 \mathrm{~min}$ during the awake state. The present data, in comparison with our previous data, indicate that this task had a considerable influence on the processing of the stimuli under deep sedation (as indexed by the P3a): it appears that the relevance which the deviants had for the subjects (and which was learned during the awake state) clearly influenced the ERPs even during a state in which participants were not able to consciously respond to the 
stimuli. Although participants were deeply sedated, attention-related brain mechanisms were still clearly activated by these relevant stimuli. Because of overlapping neurobiologic mechanisms of natural sleep and deep sedation (e.g. Tung et al., 2004), it is likely that these mechanisms are responsible for the awakening of an individual from sleep, even when auditory information has only moderate loudness.

In the ERAN blocks, no effects of music-syntactically irregular chords were observed, indicating that processes of music-syntactic analysis do not operate under deep sedation in unresponsive subjects. In the previous study from Heinke et al. (2004b), the ERAN elicited during deep sedation was, thus, most presumably due to the subgroup of responsive subjects. Note that the ERAN strongly resembles the ELAN elicited by syntactic violations during language processing (Koelsch and Friederici, 2003, see also Section 1), and that the generation of both ERAN and ELAN relies to a considerable amount on the same generators located in the inferior frontolateral cortex (in the left hemisphere often denoted as Broca's area, Friederici et al., 2000; Maess et al., 2001), and the anterior portion of the superior temporal gyrus (Friederici, 2002; Koelsch et al., 2005a). Thus, it is highly likely that not only music-syntactic processing, but also the fast and automatic processing of language-syntactic information is abolished under deep propofol sedation. This assumption is corroborated by a previous functional imaging study investigating effects of propofol sedation on auditory language processing (Heinke et al., 2004a): in that study, inferior frontolateral cortex (including Broca's area), as well as the anterior portion of the STG, was activated by a language task in responsive, but not in unresponsive subjects.

Taken together, the combined evidence suggests that the neural processes underlying the operation of the auditory sensory memory (as indexed by the MMN), and underlying attention-related processes (as indexed by the P3a and the late negativity) are still active, although strongly reduced, under deep sedation (even in subjects that are unresponsive corresponding to MOAAS 2-3). By contrast, the neural operations underlying the processing of complex, regularity-based structural information (as indexed by the ERAN) are abolished. It is possible that these different effects of propofol on different cognitive mechanisms are due to differential effects of sedative drugs on different cerebral structures (see also Heinke and Koelsch, 2005; Heinke et al., 2004b; Reinsel et al., 2000; Veselis et al., 1997).

\subsection{Recovery}

After the deep sedation, the propofol-dose was increased to induce unconsciousness (reflecting adequate anesthesia). During recovery from unconsciousness (15-45 min after the propofol-induced unconsciousness, see Section 2 and Fig. 2), a clear MMN was observed (for both frequency and timbre deviants), although propofol plasma levels were still in the sedation range (around $0.8-1.6 \mu \mathrm{g} / \mathrm{ml}$ ). This result indicates that the generation of the $\mathrm{MMN}$ is not abolished after regaining consciousness from propofolinduced anesthesia. The MMN amplitudes were, surprisingly, even comparable to the awake state, suggesting that immediately after regaining consciousness from propofolinduced anesthesia, the auditory sensory memory mechanisms (as reflected in the MMN) operate similar as during wakefulness.

The MMN amplitude did not vary between the first and the second half of the recovery period, in contrast to the propofol plasma levels that differed between beginning, middle, and end of the recovery period. This indicates that the amplitude of the $\mathrm{MMN}$ is dependent on the level of consciousness, rather than on the propofol plasma levels.

Most interestingly, no P3a was observed during the recovery period, in contrast to both the awake state and the deep sedation level (in which clear P3a potentials were observed). Note that the absence of the P3a during the recovery period cannot simply be due to the deletion of positive potentials by the (preceding) MMN potentials: strong MMN potentials were also elicited during the awake state, and during the awake state a clear P3a was nevertheless observed (also note that the peak-to-peak amplitude of the N2b-P3a complex was considerably larger in the awake state compared to the recovery period).

The P3a was not present during the recovery period, although BIS-values were clearly higher (and propofol plasma concentrations lower) than during the deep sedation (see also Table 4 and Fig. 4). This indicates that the propofol-induced loss of consciousness (induced between the level of deep sedation and the recovery period) had strong effects on the attention-related mechanisms underlying the generation of the P3a during the recovery period. That is, although participants were awake (and responded to the timbre deviants similarly as during the awake state), and although the auditory sensory memory operated quite normal (as indexed by the strong MMN), attention-related mechanisms were severely impaired in individuals after propofol-induced anesthesia.

Like the amplitude of the P3a, the amplitude of the P3b was clearly reduced during the recovery period. It is important to note that only trials in which participants responded behaviorally to the timbre deviants were included in the analysis of the ERPs elicited during both awake state and recovery, and that both reaction times and variance of reaction times did not differ between awake state and recovery. That is, despite virtually identical behavioral performance, the response-related P3b potentials strongly differed between these two levels.

The in-depth analysis of the P3b strongly suggests that the difference of the P3b amplitudes between awake state and recovery phase was not due to a greater variance of $\mathrm{P} 3 \mathrm{~b}$ latencies, neither intra- nor inter-individually. An increase in the variance of latencies would have led to an amplitude 
reduction of the $\mathrm{P} 3 \mathrm{~b}$ due to a greater jittering of $\mathrm{P} 3 \mathrm{~b}$ potentials, but this does not seem to be the case in the present data, indicating that the neural mechanisms underlying the generation of the P3b were impaired as an effect of sedation. This impairment could be due to (a) the propofol concentrations during the recovery period (see Table 4), (b) the preceding unconsciousness phase, (c) the long phase of deep sedation, or (d) a combination of those factors; this issue remains to be specified. Future studies should investigate the time course of the recovery of both $\mathrm{P} 3 \mathrm{a}$ and $\mathrm{P} 3 \mathrm{~b}$, because the recovery of $\mathrm{P} 3 \mathrm{a}$ and $\mathrm{P} 3 \mathrm{~b}$ could provide useful indices for complete cognitive recovery from anesthesia.

\section{Acknowledgements}

The work was financially supported by the Max Planck Institute for Human Cognitive and Brain Sciences, Leipzig, Germany, the Department of Anesthesiology and Intensive Care Therapy, University of Leipzig, and the Verein zur Förderung der Forschung und Fortbildung in der Anästhesiologie, Intensiv-, Schmerz- und Notfalltherapie e.V., Leipzig, Germany.

The authors thank Kristiane Werrmann for EEG data acquisition, and Barbara Vetter for propofol plasma level measurements.

\section{References}

Alain C, Woods DL, Knight RT. A distributed cortical network for auditory sensory memory in humans. Brain Res 1998;812:23-37.

Alho K, Woods DL, Algazi A, Knight RT, Näätänen R. Lesions of frontal cortex diminish the auditory mismatch negativity. Clin Neurophys 1994;91:353-62.

Alho K, Escera C, Diaz R, Yago E, Serra J. Effects of involuntary auditory attention on visual task performance and brain activity. Neuroreport 1997;8(15):3233-7.

Billard V, Gambus P, Chamoun N, Stanski D, Shafer S. A comparison of spectral edge, delta power, and bispectral index as EEG measures of alfentanil, propofol, and midazolam drug effect. Clin Pharmacol Ther 1997;61(1):45-58.

Chernik D, Gillings D, Laine H, Hendler J, Silver J, Davidson A, Schwam E, Siegel J. Validity and reliability of the observer's assessment of alertness/sedation scale: study with intravenous midazolam. J Clin Psychopharmacol 1990;10:244-51.

Doeller C, Opitz B, Mecklinger A, Krick C, Reith W, Schröger E. Prefrontal cortex involvement in preattentive auditory deviance detection: neuroimaging and electrophysiological evidence. Neuroimage 2003;20(2):1270-82.

Donchin E. Event-related potentials: a tool in the study of human information processing. In: Begleiter $\mathrm{H}$, editor. Evoked brain potentials and behavior. New York: Plenum Press; 1979. p. 13-75.

Escera C, Alho K, Schröger E, Winkler I. Involuntary attention and distractibility as evaluated with event-related brain potentials. Audiol Neuro-Otol 2000;5(3-4):151-66.

Friederici AD. Towards a neural basis of auditory sentence processing. Trends Cogn Sci 2002;6(2):78-84.
Friederici AD, Wang Y, Herrmann CS, Maess B, Oertel U. Localisation of early syntactic processes in frontal and temporal cortical areas: an MEG study. Hum Brain Mapp 2000;11:1-11.

Ghoneim M. Awareness during anesthesia. Anesthesiology 2000;92(2): 597-602.

Heinke W, Koelsch S. The effects of anesthetics on brain activity and cognitive function. Curr Opin Anaesthesiol 2005;18:625-31.

Heinke W, Fiebach C, Schwarzbauer C, Meyer M, Olthoff D, Alter K. Sequential effects of propofol on functional brain activation induced by auditory language processing: an event-related functional magnetic resonance imaging study. Br J Anaesth 2004a;92(5):641-50.

Heinke W, Kenntner R, Gunter TC, Sammler D, Olthoff D, Koelsch S. Differential effects of increasing propofol sedation on frontal and temporal cortices: an ERP study. Anesthesiology 2004b;100: $617-25$.

Janata P, Grafton ST. Swinging in the brain: shared neural substrates for behaviors related to sequencing and music. Nat Neurosci 2003;6(7): $682-7$.

Johansen J, Sebel P. Development and clinical application of electroencephalographic bispectrum monitoring. Anesthesiology 2000;93(5): 1336-44.

Koelsch S. Neural substrates of processing syntax and semantics in music. Curr Opin Neurobiol 2005;15:1-6.

Koelsch S, Friederici AD. Towards the neural basis of processing structure in music: comparative results of different neurophysiological investigation methods (EEG, MEG, fMRI). Ann NY Acad Sci 2003;999: $15-27$.

Koelsch S, Siebel WA. Towards a neural basis of music perception. Trends Cogn Sci 2005;9(12):578-84.

Koelsch S, Fritz T, Schluze K, Alsop D, Schlaug G. Adults and children processing music: an fMRI study. Neuroimage 2005a;25: $1068-76$.

Koelsch S, Gunter TC, Wittfoth M, Sammler D. Interaction between syntax processing in language and in music: an ERP study. J Cogn Neurosci 2005b;17:1565-79.

Maess B, Koelsch S, Gunter TC, Friederici AD. Musical syntax is processed in the area of Broca: an MEG-study. Nat Neurosci 2001;4(5): $540-5$.

Näätänen R. Attention and brain function. Hillsdale, NJ: Erlbaum; 1992.

Nääanen R. The perception of speech sounds by the human brain as reflected by the mismatch negativity (MMN) and its magnetic equivalent (MMNm). Psychophysiology 2001;38:1-21.

Opitz B, Rinne T, Mecklinger A, von Cramon DY, Schröger E. Differential contribution of frontal and temporal cortices to auditory chenage detection: fMRI and ERP results. Neuroimage 2002;15:167-74.

Patel AD. Language, music, syntax and the brain. Nat Neurosci 2003;6(7): 674-81.

Patel AD. The relationship of music to the melody of speech and to syntactic processing disorders in aphasia. Ann NY Acad Sci 2005;1060: 717-33.

Patel AD, Gibson E, Ratner J, Besson M, Holcomb P. Processing syntactic relations in language and music: an event-related potential study. J Cogn Neurosci 1998;10(6):717-33.

Reinsel R, Veselis R, Dnistrian A, Feshchenko V, Beattie B, Duff M. Midazolam decreases cerebral blood flow in the left prefrontal cortex in a dose-dependent fashion. Int J Neuropsychopharmacol 2000;3:117-27.

Rinne T, Alho K, Ilmoniemi RJ, Virtanen J, Näätänen R. Separate time behaviors of the temporal and frontal mismatch negativity sources. Neuroimage 2000;12:14-19.

Rosow C, Manberg P. Bispectral index monitoring. Anesthesiol Clin North Am 2001;19(4):947-66.

Rundshagen I, Schnabel K, Schulte am Esch J. Impaired explicit memory after recovery from propofol/sufentanil anaesthesia is related to changes in the midlatency auditory evoked response. Br J Anaesth 2002a;89:376-81. 
Rundshagen I, Schnabel K, Schulte am Esch J. Midlatency auditory evoked potentials do not allow the prediction of recovery from general anesthesia with isoflurane. Can J Anaesth 2002b;49:361-8.

Schröger E. Measurement and interpretation of the mismatch negativity (MMN). Behav Res Methods Instrum Comput 1998;30:131-45.

Schröger E, Wolff C. Attentional orienting and reorienting is indicated by human event-related brain potentials. NeuroReport 1998;9(15): $3355-8$.

Simpson TP, Manara AR, Kane NM, Barton RL, Rowlands CA, Butler SR. Effect of propofol anaesthesia on the event-related potential mismatch negativity and the auditory-evoked potential N1. Br J Anaesth 2002;89: $382-8$.
Tung A, Bergmann BM, Herrera S, Cao D, Mendelson WB. Recovery from sleep deprivation occurs during propofol anesthesia. Anesthesiology 2004;100(6):1419-26.

Veselis RA, Reinsel RA, Beattie B, Mawlawi O, Feshchenko VA, DiResta G, Larson S, Blasberg R. Midazolam changes cerebral blood flow in discrete brain regions: an h2(15)o positron emission tomography study. Anesthesiology 1997;87(5):1106-17.

Veselis RA, Reinsel RA, Feshchenko VA. Drug-induced amnesia is a separate phenomenon from sedation. Anesthesiology 2001;95:896-907. Yppärilla H, Karhu J, Westerén-Punnonen S, Musialowicz T, Partanen J. Evidence of auditory processing during postoperative propofol sedation. Clin Neurophysiol 2002;113:1357-64. 